\title{
Half Century of Gold Price: Regime-Switching and Forecasting Framework
}

\author{
Nguyen Bao Anh ${ }^{1} \&$ Yiqiang Q. Zhao ${ }^{2}$ \\ ${ }^{1}$ Dong Nai Technology University, Vietnam \\ ${ }^{2}$ Carleton University, Canada \\ Correspondence, Nguyen Bao Anh, Dong Nai Technology University, Vietnam; Carleton University, Canada. E-mail: \\ baoanh.nguyen@carleton.ca \\ Received: October 6, 2020 \\ Accepted: December 12, $2020 \quad$ Online Published: January 11, 2021 \\ doi:10.5430/ijfr.v12n3p1 \\ URL: https://doi.org/10.5430/ijfr.v12n3p1
}

\begin{abstract}
This paper studies the history of gold price in the international context using Markov-switching models. The literature surrounding the Markov-switching model is reviewed from the earliest iterations of Hamilton to recent developments. We show applicability of Markov stochastic process in forecasting commodity prices; in particular, the gold spot price. The research imposes the features of Markov regime-switching models, considering gold as a financial asset to offer a comprehensive methodology for forecasting commodity price. The paper discovers that applying Markov regime-switching could significantly improve the forecast abilities in commodity prices. Analysis of the model outcome indicates that the abnormal increases of gold price in history always resulted from special economic conditions. This study makes a novel contribution to the field by demonstrating that the impact of CPI change to gold price is subject to the regimes, which is more sophisticated than what has been commonly accepted in economics literature to date.
\end{abstract}

Keywords: Markov-switching model, gold price, MS auto-regression, regime-switching

\section{Introduction}

\subsection{Economic Conditions and Questions on Regimes}

In the first half of 2020, we observed extraordinary socio-economic situations and indicators of economic development. The economic growth rate of Canada was projected to be between 1.6 to $1.8 \%$ in 2020 (Source: Conference Board of Canada https://www.conferenceboard.ca/topics/economics) but turned out to have an actual drop to annualized $-2.1 \%$ in the first quarter (Source: Statistics Canada https://www150.statcan.gc.ca).

The Canadian oil patch faced the worst crisis in its history due to a confluence of factors, including a collapse in demand for all types of fuel caused by the Covid-19 shut down, and an unprecedented oil surplus due to the failure of the Organization of Petroleum Exporting Countries and their allies to reach an agreement on new production quotas until late April. The negative oil price in April, which shocked the statisticians, economists, and even computer application programmers, those who set positive value constraints for transaction price in software.

The appreciation of precious metals and stock indexes was also observed. The gold spot price reached 1,715.90 USD/ounce, which is the highest price since the end of 2015 where it stood at 1,060.00 USD/ounce. Supposing that one bought and held gold from that year, it would be possible to see a gain of $70 \%$ from the initial investment. The Bank of America projected that the Covid-19 pandemic would drive gold to a lofty record by October 2021. The average gold price in 2020 would reach 1,695 USD/ounce before the soaring demand pushes it to 2,063 USD/ounce the following year (Source: https://www.msn.com/en-au/money/markets/). Above all, gold and other natural resource commodities in normal economic circumstances remain volatile as an individual asset. In a larger perspective, the downturn has affected all aspects of Canada's economy, though some sectors have been particularly hard hit in a manner equivalent to the 2008-09 financial crisis.

The interesting question emerging from these concerns how statisticians, economists, and researchers, among others, can detect the different behaviors of socio-economic data in times of crisis. Naturally, we consider the potential methodologies that could learn and analyze the changes of states in the past, apply the different parameters in different periods and estimate the possibility to switch between states in the forecast models. Markov Regime-switching would give us a possibility to detect different regimes throughout the history.

In times of crisis, while stock markets crash in general, some physical assets turn out to be on the top of the list of 
items that investors would like to hold for maintaining their value in their portfolios. Precious metals such as gold, silver, and platinum are always in an investors mind. There is the obvious implication that the stocks of precious metal miners and producers would appreciate because their products bring increased profits. Another advantage of gold stock is that they are subject to depreciation and appreciation like other stocks. So, if there is a positive change in stock values, driven by either an increase in gold prices or market shocks, gold mining stocks go up. Because these appreciate like stocks, the extent of appreciation can be even more significant. That increases the justification for researchers and scholars to develop the application of Markov regime-switching in forecasting models on the price and returns of gold in the international or in a particular market.

\subsection{Background}

First introduced in 1989 in the seminal work of Hamilton, Markov switching models are a well-studied family of models in probability theory imposing time-varying parameters in different periods of time, known as state or regime-specific values. Statistically, this time-variation is governed by a discrete-valued latent stochastic process in the past. Specifically, as a feature of the Markov chain, the current value of the state is determined only by its value from the immediate previous period, and the transition probability matrix known as a system of dynamic transformation. The transition probability matrix regulates the properties of the Markov process by determining the probability that each of the states can be visited in the next period given the current state. The estimates of the transition probability entries and the matrix can be evaluated using filtering and smoothing methods as well as the estimation of state-specific parameters. These two features make it possible to have improved interpretations of the parameters associated with specific regimes combined with the corresponding regime probabilities, as well as for improved forecasting of performance based on persistent regimes and parameters characterizing them.

The characteristics of the physical gold commodity are high liquidity, consumption, and convenient value storage. It is also a homogenous good which can be easily converted in transactions or by contracts. Therefore, not surprisingly, gold has a special position among other precious metals as an investment item and a type of currency to prevent inflation. Previous studies in the literature provide limited evidence that the fluctuation of the gold market is affected by the same macroeconomic factors as is the case for other metals (in the paper Trends of Base Metals Prices (Note 1), we showed that macroeconomic factors such as technological progress, resource scarcity, natural resource taxes, and interest rates are the determinants of base metal price). The risk-mitigating features of gold have been discussed adequately in the literature, which has noted the increasingly important role played by gold as a hedge against inflation.

Practically, gold price forecasting is performed by an average prediction of a diverse panel of experts and gold market analysts. Their assessments of gold price trends are based on a variety of methods including expert technical analysis, market fundamentals, current market sentiment, and an analysis of global economic and political events (Note 2).

In Figure 1 below we can see how the gold spot price in major currencies changes throughout history (Note 3). In some periods, the price of gold in CAD and USD are quite close (from 2005 to 2013), while in others (from 2015 to 2020 or from 1980 to 1990) there are a gap between their co-movements.

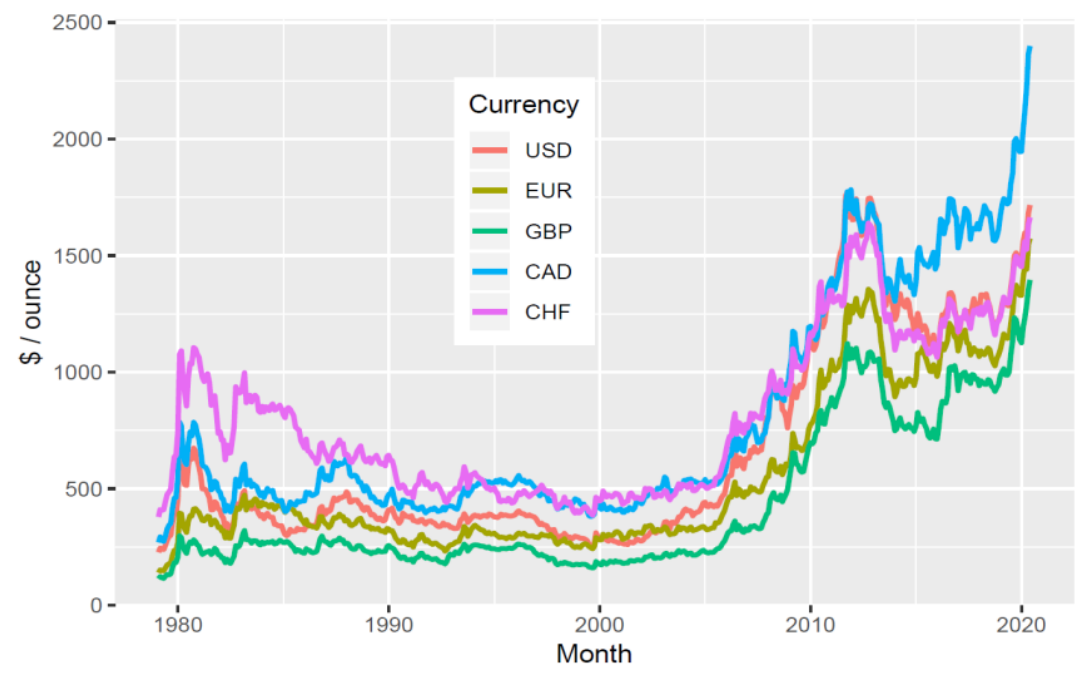

Figure 1. Gold price in major currencies 
Gold price moves in the opposite direction of inflation. This reflects the basic function of gold as an asset insofar as it stores value against inflation. Through a macroeconomics foundation, the relationship between a pair of currencies, or in other words, the exchange rate, depends on six factors: Differentials in Inflation, Differentials in Interest Rates, Current Account Deficits, Public Debt, Terms of Trade, and Strength of Economic Performance.

All the factors and relationships among them impacting hundreds of economies around the world suggest that it is not realistic to build a model capable of accounting for such an extensive number of determinants. In the present document we use the gold prices in another currency (USD) and Consumer Price index to predict the price in CAD. This model reflects the reality that Canada is not a major consumer of gold and that the gold price in CAD depends on the tendencies of markets in other economies.

The study of gold price and returns benefits investors of gold stocks, except for physical gold. When an investor selects a gold stock, he or she is not buying an amount of physical gold, gold bullion or gold coins. Consider, for example, mining stocks. The benefit is quick gains if the mining stocks perform well as for-profit economic entities. In a relative interaction, physical gold is better suited for anti-inflation investments. If we are investing in miners, we are investing in gold mining stocks, and therefore in particular companies. We assume that they are well managed as the standard in the industry. Indeed, one stock presents for the financial health or profit of one particular company. This research also refers to the index of gold stock in NYSE to study and illustrate the implication.

It may be of interest to commodity investors and stock exchanges that the price of gold would impact the indices of gold stock. If gold price is high, then the gold mines would gain better profits and hence, the stock price would increase. Figure 2 shows the co-movement of gold price in USD and the HUI, XAU indices - the two most well-known gold indices in the financial markets (Note 4).

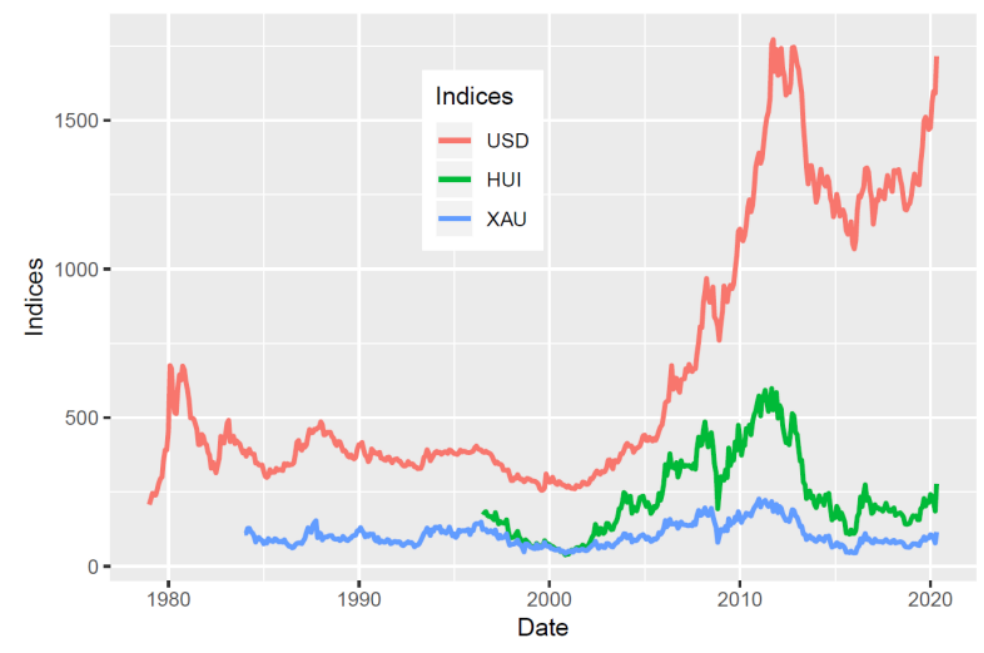

Figure 2. Indices of gold stocks vs. gold price in USD

\subsection{Main Contributions}

This study involves literatures on Statistics of Financial Markets: Markov Chain, Auto-regression, and Regime-switching models. We illustrate applications of Markov regime-switching models in an analysis of historical gold price, using the availability of data from the World Gold Council. The following are the major contributions of the work to literature: It contributes to the understanding of the development in Markov-switching Models studies, and their applications in forecasting works. The research provides a novel contribution, according to our best knowledge, introducing and presenting a forecasting model for gold spot price using the Markov Regime-switching auto-regression and the selected exogenous variables: USD value and CPI change. We discover that, changes in CPI impact gold price in a sophisticated manner, subject to the regimes. In doing so we show that applying Markov regime-switching could significantly improve the forecast abilities in commodity prices. The study also analyzes the periods of time that gold price switches; abnormal increases of gold price always result from special economic conditions. The main results are interpreted and presented, together with proposals for potential future research. The study offers novel results for forecasting a particular type of asset in financial theory or fundamental statistics. 


\subsection{Organization}

The remaining portion of the paper is organized as follows: Part 2 reviews theoretical background, concepts, and methods for estimating the models' parameters. We systematize the literature of Markov regime-switching models and summarize the development of studies in the field. Part 3 compares popular models and our MS auto-regression model on gold price forecasting works. Part 4 concludes and discusses potential research to fully capture these concepts in future research. The Appendices list abbreviations and symbols to be used in the paper.

\section{Literature Review}

\subsection{Markov Chain \& Regime-Switching}

Regime-switching models are the models that can characterize time series properties in different regimes. Models in which the switching among regimes occurs stochastically according to a Markov process are called Markov regime-switching models or Markov switching models (MS, MSM) for short.

We start with the definitions of basic terms and concepts that will be used to develop a unified approach for building our Markov switching model. Only the concepts of relevant terms are defined in this section because there are sufficient definitions in textbooks and in the literature.

\subsubsection{Definitions and Concepts}

In the below definitions, the state of a stochastic process $S_{t}, t=0,1, \ldots$ at time $t$ is the value of $S_{t}$, denoted by $s_{t}$, indexed in its value space by $i, j$.

\section{Definition 2.1: Markov Chain}

A discrete time Markov chain is defined as a stochastic process $S_{t}, t=0,1, \ldots$ such that, at all time $t$, the probability of the future state $S_{t+1}$, is only dependent on the present state and independent of the past states, that is:

$$
P\left(S_{t+1}=j \mid S_{t}=i_{t}, \ldots, S_{1}=i_{1}, S_{0}=i_{0}\right)=P\left(S_{t+1}=j \mid S_{t}=i_{t}\right)
$$

This probability, denoted by $p_{i j}$, is the transition probability from $i$ to $j$, the probability that the state will next be $j$ when the current state is $i$.

\section{Definition 2.2: Transition Probability Matrix}

$$
\boldsymbol{P}_{\boldsymbol{k} \boldsymbol{k}}=\left[\begin{array}{ccc}
p_{1,1} & \cdots & p_{1, k} \\
\vdots & \ddots & \vdots \\
p_{k, 1} & \cdots & p_{k, k}
\end{array}\right]
$$

The Transition Probability Matrix of $k$ states is a matrix whose $(i, j)$-entry is the transition probability to state $j$ given the previous state is $i$.

The multi-step transition probabilities can be calculated recursively. In general, the $n$-step transition probability is

$$
P\left\{S_{t+n}=j \mid S_{t}=i\right\}=P_{i j}^{n}
$$

This leads to the formula for calculating the multi-step transition probability, from state $i$ to state $j$ via state $k$, known as Chapman-Kolmogorov equation.

\section{Theorem 2.1: Chapman-Kolmogorov equation}

$$
P_{i j}^{m+n}=\sum_{k=1}^{N} P_{i k}^{n} P_{k j}^{m} \quad i, j=1,2, \ldots N
$$

The following properties of stochastic matrices hold:

\section{Lemma: Properties of Transition Probability Matrix}

- All entries take values in the range [0,1], and each row sums to 1 .

- The Markov chain is homogeneous in the sense that the $(i, j)$-entry of the transition probability matrix gives the probability that the process moves from the $i^{\text {th }}$ state to the $j^{\text {th }}$ state during the next step of the process, independent of time.

- The probability vector after $n$ steps of a Markov chain is $P^{n} \vec{v}$, where $\vec{v}$ is the initial probability vector and $P$ is the transition probability matrix. A limit vector for a Markov chain is always a fixed point (a vector $\vec{v}$ such that $P \vec{v}=\vec{v}$ ).

Example: Assuming that we have two regimes (or states), representing the normal condition of the economy and the economy in crisis time. Then, the transition matrix is given by: 


$$
\boldsymbol{P}_{\boldsymbol{k} \boldsymbol{k}}=\left|\begin{array}{ll}
p_{1,1} & p_{1,2} \\
p_{2,1} & p_{2,2}
\end{array}\right|
$$

where $p_{1,1}+p_{1,2}=1$ and $p_{2,1}+p_{2,2}=1$, and

$$
\boldsymbol{P}_{\boldsymbol{k} \boldsymbol{k}}=\left|\begin{array}{ll}
P_{\text {Normal at } t+1 \mid \text { Normal at } t} & P_{\text {Crisis at } t+1 \mid \text { Normal at } t} \\
P_{\text {Normal at } t+1 \mid \text { Crisis at } t} & P_{\text {Crisis at } t+1 \mid \text { Crisis at } t}
\end{array}\right|
$$

As an illustration, we would follow these steps to compute the transition matrix to model the transition between the normal condition and crisis time: Suppose that there are 200 observations, of which 125 observations show that they keep remaining in normal condition, 5 observations exhibit the shift from normal condition to crisis time, 60 observations move from crisis to normal, and 10 observations show that they remain in crisis given that the previous period of time is in crisis.

The frequencies, or the numbers of observations for the different transitions:

$$
\boldsymbol{O}_{k \boldsymbol{k}}=\left|\begin{array}{cc}
125 & 05 \\
60 & 10
\end{array}\right|
$$

We then normalize the rows such that their sum is equal to 1:

$$
\boldsymbol{P}_{\boldsymbol{k} \boldsymbol{k}}=\left|\begin{array}{ll}
0.962 & 0.038 \\
0.875 & 0.143
\end{array}\right|
$$

In this example, the probability of switching from regime 1 normal condition to regime 2 crisis time is $3.8 \%$, the probability of staying in crisis is $14.3 \%$ if the previous state is crisis.

\section{Definition 2.3: Conditional, Filtered, and Smoothing Probabilities}

Conditional probability is the probability that $S_{t}$ take the value $s_{t}$ based on the information prior to time $t$ (i.e $t-1)$, Filtering probability is the probability that $S_{t}$ take the value $s_{t}$ based on the information in the past and current information, and Smoothing probability is the probability that $S_{t}$ take the value $s_{t}$ based on the full sample information.

That is

$$
\begin{aligned}
P_{\text {conditional }} & =P\left(S_{t}=s_{t} \mid \Omega_{t-1}\right) \\
P_{\text {filtered }} & =P\left(S_{t}=s_{t} \mid \Omega_{t}\right) \\
P_{\text {smoothing }} & =P\left(S_{t}=s_{t} \mid \Omega_{T}\right)
\end{aligned}
$$

In this paper we study estimation methods using the maximum likelihood function. To assess the likelihood of the state variable $S_{t}$, it is critical to evaluate its optimal forecasts (conditional expectations) of $S_{t}=S_{t}$, based on different information sets. These forecasts include the predictions of $P_{\text {conditional }}, P_{\text {filtered }}$, and $P_{\text {smoothing }}$.

To estimate the parameters of the Markov-switching model we consider the joint conditional probability of each future state, as a function of the joint conditional probabilities of current states and the transition probabilities.

\section{Definition 2.4: Filtering Process}

A filtering process, with a system of dynamic transformation, is a process where the input is the conditional probabilities of the current states. The system of dynamic transformation is the transition probability matrix. The output is the conditional probability of future states.

That is

$$
P\left(S_{t}=s_{t} \mid y_{t-1}\right)=P\left(S_{t}=s_{t} \mid S_{t-1}=s_{t-1}\right) \times P\left(S_{t-1}=s_{t-1} \mid y_{t-1}\right)
$$

where the output $P\left(S_{t}=s_{t} \mid y_{t-1}\right)$ is the conditional probability of being in state $s_{t}$ given information at $t-1$. The input $P\left(S_{t}=s_{t} \mid S_{t-1}=s_{t-1}\right)$ stands for the conditional probabilities of the current states. The system of dynamic transformation $P\left(S_{t-1}=s_{t-1} \mid y_{t-1}\right)$ is the transition probability matrix.

\subsubsection{Estimating Parameters}

The parameters of the system can be estimated by solving the maximizing likelihood function problem. The likelihood function is the probability density of the data, viewed as a function of the parameters, takes the dataset as a given. The conditional likelihood function is the product of conditional probability density functions. Consider a two-state model with a Markov chain process: $y_{t}=\sum_{i=1}^{k} I_{i} \vec{\theta}_{i}+\varepsilon_{t}$, where $i \in[1,2], I_{i}=1$ in state $i$ and 0 
otherwise, and $\varepsilon_{t}$ is a white noise residual. We want to evaluate the conditional probability $P\left(S_{t}=s_{t} \mid y_{t-1}\right)$, which is the probability of being in state $s_{t}$ given the information available at time $t-1$. Using the transition probability and its properties, this task is quite possible by the filtering process and equation (4). Eventually we are able to estimate the filtering probability, the probability of being in state $s_{t}, P\left(S_{t} \mid y_{t}\right)$ by imposing the information we know at time $t$.

The joint density distribution of $y_{t}$ and $S_{t}$

$$
\begin{aligned}
& f\left(y_{t}, S_{t}=s_{t} \mid y_{t-1}\right)=f\left(y_{t} \mid S_{t}=s_{t}, y_{t-1}\right) \times P\left(S_{t}=s_{t} \mid y_{t-1}\right) \\
& =f\left(y_{t} \mid S_{t}=s_{t}, y_{t-1}\right) \times P\left(S_{t}=s_{t} \mid S_{t-1}=s_{t-1}\right) \\
& \times P\left(S_{t-1}=s_{t-1} \mid y_{t-1}\right)
\end{aligned}
$$

The density distribution of $y_{t}$

$$
f\left(y_{t} \mid y_{t-1}\right)=\sum_{s_{t}=1}^{2} f\left(y_{t}, S_{t}=s_{t} \mid y_{t-1}\right)
$$

The updated joint probability of $y_{t}$ and $S_{t}$ is

$$
P\left(S_{t}=s_{t} \mid y_{t}\right)=\frac{f\left(y_{t}, S_{t}=s_{t} \mid y_{t-1}\right)}{f\left(y_{t} \mid y_{t-1}\right)}
$$

In reality we are facing many forecasting problems that have more than two states. They can be solved generally by an auto-regressive intergrated moving average with exogenous variables model where the data series is auto-regressive of order $p$ with exogenous variables $x_{i t}$ (ARIMAX). Let $\Omega_{t-1}$ denote the available information set $x_{k}, y_{k}, k=t-1, t-2, \ldots, t-p$. The strategy to estimate the model's parameters is well studied in the literature (for example, Hamilton, 1989, and Wang, 2002). Considering the data series as a Markov chain process, then the joint conditional probability of the current state and $p$ previous states is

$$
P\left(S_{t}=s_{t}, S_{t-1}=s_{t-1}, \ldots, S_{t-p}=s_{t-p} \mid \Omega_{t-1}\right)
$$

The joint density distribution of $y_{t}$ and $S_{t}$ is

$$
\begin{aligned}
& g\left(y_{t}, S_{t}=s_{t}, S_{t-1}=s_{t-1}, \ldots, S_{t-p-1}=s_{t-p-1} \mid \Omega_{t-1}\right) \\
& =g\left(y_{t}, S_{t}=s_{t}, S_{t-1}=s_{t-1}, \ldots, S_{t-p-1}=s_{t-p-1}, \Omega_{t-1}\right) \\
& \times P\left(S_{t}=s_{t}, S_{t-1}=s_{t-1}, \ldots, S_{t-p-1}=s_{t-p-1} \mid \Omega_{t-1}\right) \\
& =g\left(y_{t}, S_{t}=s_{t}, S_{t-1}=s_{t-1}, \ldots, S_{t-p-1}=s_{t-p-1}, \Omega_{t-1}\right) \\
& \times P\left(S_{t}=s_{t} \mid S_{t-1}=s_{t-1}\right) \times P\left(S_{t-1}=s_{t-1}, \ldots, S_{t-p-1}=s_{t-p-1} \mid \Omega_{t-1}\right)
\end{aligned}
$$

The density distribution of $y_{t}$

$$
g\left(y_{t} \mid \Omega_{t-1}\right)=\sum_{s_{t}=1}^{k} \sum_{s_{t-1}=1}^{k} \ldots \sum_{s_{t-p}=1}^{k} f\left(y_{t}, S_{t}=s_{t}, S_{t-1}=s_{t-1}, \ldots, S_{t-p-1}=s_{t-p-1}, \Omega_{t-1}\right)
$$

The updated joint probability of $y_{t}$ and $S_{t}$ at time $t$

$$
P\left(S_{t}=s_{t} \mid y_{t}\right)=\frac{g\left(y_{t}, S_{t}=s_{t}, S_{t-1}=s_{\left.t-1, \ldots, S_{t-p-1}=s_{t-p-1} \mid \Omega_{t-1}\right)}\right.}{g\left(y_{t} \mid \Omega_{t-1}\right)}
$$

The summation over the states at lag $p$ is just the output of the filter

$$
P\left(S_{t}=s_{t}, S_{t-1}=s_{t-1}, \ldots, S_{t-p}=s_{t-p} \mid \Omega_{t}\right)=\sum_{s_{t-p-1}=1}^{N} P\left(S_{t}=s_{t}, S_{t-1}=s_{t-1}, \ldots, S_{t-p-1}=s_{t-p-1} \mid \Omega_{t}\right)
$$

The probability of the states at time $t$ is obtained

$$
P\left(S_{t}=s_{t} \mid \Omega_{t}\right)=\sum_{s_{t}=1}^{N} \sum_{s_{t-1}=1}^{N} \cdots \sum_{s_{t-p}=1}^{N} P\left(S_{t}=s_{t}, \ldots, S_{t-p-1}=s_{t-p-1} \mid \Omega_{t}\right)
$$

The likelihood function, where $\vec{\theta}$ denotes the vector of the set of parameters,

$$
L(\vec{\theta})=\prod_{t=1}^{T} g\left(y_{t} \mid \Omega_{t-1} ; \vec{\theta}\right)
$$

Logarithmizing the likelihood function yields

$$
\mathcal{L}(\vec{\theta})=\sum_{t=1}^{T} \log g\left(y_{t} \mid \Omega_{t-1} ; \vec{\theta}\right)
$$

Solving the problems of maximizing the likelihood function, yields the MLE for parameters $\vec{\theta}$. For simplicity we illustrate the two-state Markov chain process of equation $y_{t}=\sum_{i=1}^{k} I_{i} * \vec{\theta}_{i}+\varepsilon_{t}: i=1,2$. Let 
$P_{t L}(1)=P\left(S_{t-1}=1 \mid y_{t-1}\right)$ and $P_{t L}(2)=P\left(S_{t-1}=2 \mid y_{t-1}\right)$. Assuming that the residual is normally distributed, then the maximum log-likelihood function is

$$
\begin{aligned}
& L(\vec{\theta})=\sum_{t=1}^{T} f\left(y_{t} \mid \Omega_{t-1} ; \vec{\theta}\right) \\
& =\sum_{t=1}^{T} \sum_{s_{t}=1}^{2} f\left(y_{t} \mid S_{t}=s_{t}, y_{t-1} ; \vec{\theta}\right) \times P\left(S_{t}=s_{t} \mid y_{t-1}\right) \\
& =\sum_{t=1}^{T} \sum_{s_{t}=1}^{2} \sum_{s_{t-1}=1}^{2}\left\{f\left(y_{t} \mid S_{t}=s_{t}, y_{t-1} ; \vec{\theta}\right)\right. \\
& \left.\times P\left(S_{t}=s_{t} \mid S_{t-1}=s_{t-1}\right) \times P\left(S_{t-1}=s_{t-1} \mid y_{t-1}\right)\right\} \\
& =\sum_{t=1}^{T}\left\{\frac{1}{\sqrt{2 \pi \sigma}} \exp \left[\frac{-\left(y_{t}-\mu_{1}\right)^{2}}{2 \sigma^{2}}\right] \times\left[p_{11} \times P_{1 L}(1)+p_{21} \times P_{t L}(2)\right]\right. \\
& \left.+\frac{1}{\sqrt{2 \pi \sigma}} \exp \left[\frac{-\left(y_{t}-\mu_{2}\right)^{2}}{2 \sigma^{2}}\right] \times\left[p_{21} \times P_{t L}(1)+p_{22} \times P_{t L}(2)\right]\right\}
\end{aligned}
$$

where $\mu_{1}, \mu_{2}$ are the mean values of $y_{t}$ in the two states.

Smoothing process considers the whole data available to estimate the parameters. At time $t$ we have a set of information about what happened until the last period $t-1$, which we base our estimate for state $S_{t}$. Whenever the new information arrives, we take the chance to update the estimated $S_{t}$. We can also use the information at time $t+1$ or further in the future to smooth the data series. The smoothing process revises $P\left(S_{t}=s_{t} \mid \Omega_{t}\right)$ to reflect the most accurate estimate possible. Denote the revised smoothing probability by $P\left(S_{t}=s_{t} \mid \Omega_{T}\right)$

$$
\begin{aligned}
& P\left(S_{t-p}, \ldots, S_{t}, S_{t+1} \mid \Omega_{T}\right)=P\left(S_{t-p+1}, \ldots, S_{t}, S_{t+1} \mid \Omega_{T}\right) \times P\left(S_{t-p} \mid S_{t-p+1}, \ldots, S_{t+1}, \Omega_{T}\right) \\
& =P\left(S_{t-p}, \ldots, S_{t+1} \mid \Omega_{T}\right)=P\left(S_{t-p+}, S_{t-p}, \ldots, S_{t+1} \mid \Omega_{T}\right) \times P\left(S_{t-p} \mid S_{t-p+1}, \ldots, S_{t+1}, \Omega_{t}\right) \\
& =\frac{\left.S_{t-p+1}, \ldots, S_{t}, S_{t+1} \mid \Omega_{T}\right) \times P\left(S_{t-p}, S_{t-p+1}, \ldots, S_{t}, S_{t+1}, \Omega_{t}\right)}{P\left(S_{t-p+1}, S_{t-p, \ldots,}, S_{t+1} \mid \Omega_{t}\right)} \\
& =\frac{\left.S_{t-p+1}, \ldots, S_{t}, S_{t+1} \mid \Omega_{T}\right) \times P\left(S_{t-p}, S_{t-p+1}, \ldots, S_{t}, S_{t+1}, \Omega_{t}\right) \times P\left(S_{t+1} \mid S_{t}\right.}{P\left(S_{t-p+1}, S_{t-p}, \ldots, S_{t}, S_{t+1} \mid \Omega_{t}\right)}
\end{aligned}
$$

Assuming that the probability density function remains unchanged, or approximating over the two consequent periods $t-p$ to $t-p+1$, that is

$$
f\left(y_{t+1}, \Omega_{T t} \mid S_{t-p}, S_{t-p+1}, \ldots, S_{t}, S_{t+1}, \Omega_{t}\right) \approx f\left(y_{t+1}, \Omega_{T t} \mid S_{t-p+1}, \ldots, S_{t}, S_{t+1}, \Omega_{t}\right)
$$

where $\Omega_{T t}=\Omega_{T}-\Omega_{t}$ then the second equality holds, meaning that we will have

$$
P\left(S_{t-p} \mid S_{t-p+1}, \ldots, S_{t}, S_{t+1}, \Omega_{T}\right)=P\left(S_{t-p} \mid S_{t-p+1}, \ldots, S_{t}, S_{t+1}, \Omega_{t}\right)
$$

Now summing over $S_{t+1}=1,2, \ldots N$ we obtain the probabilities of visiting $s_{t-p}, \ldots, s_{t}$, given the whole sample:

$$
P\left(S_{t-p}, \ldots, S_{t} \mid \Omega_{T}\right)=\sum_{S_{t+1}=1}^{N} P\left(S_{t-p}, \ldots, S_{t}, S_{t+1} \mid \Omega_{T}\right)
$$

These are the smoothed states given no serial correlation. In the cases where there are no lags $y_{t}$ involved, we obtain the smoothing probability

$$
P\left(S_{t} \mid \Omega_{T}\right)=\sum_{S_{t}=1}^{N} \sum_{S_{t-1}=1}^{N} \ldots \sum_{S_{t-p}=1}^{N} P\left(S_{t}, \ldots, S_{t-p} \mid \Omega_{T}\right)
$$

That implies that $P\left(S_{t} \mid \Omega_{T}\right)$ is equal the summation over all probabilities in states $S_{t}$ to $S_{t-p}$ in the whole space of state values.

\subsection{Empirical Literature Review}

We can divide the literature of Markov-switching models into two periods: from its introduction until the year 2000, it played a major role in solving problems in business cycle and financial research; after 2000, interest in switching models became broader with applications in technical issues and complex models of regime-switching.

Within the scope of this research, we review some notable studies and applications.

\subsubsection{Literature of the Field From 1989 to 2000}

Initially originating from the publications of Hamilton $(1989,1990)$, Markov switching models have drawn much attention in technical, financial, and economic data series analysis. In the long run there are changes in business environment, behaviors of customers or even the natural conditions. Collectively, these movements can be defined as 
regime shifts, where the parameters of the process changed. The Markov switching model exhibits more than one state or regime; it can present the dynamic process of the studied variables and shows us how these variables are evolving over times, and hence, gives us the perspective of the process in the future. Probably most of the applied research is in business cycles where recent studies are still burgeoning. In the time of digital electronic devices booming with the availability of large datasets, the Markov switching model has been widely applied because there are more chances to discover different regimes in historical data (Hamilton, 1989).

Sola, Martin and Driffill, and Edward (1994), tested the term structure of interest rates using a stationary vector auto-regression with regime switching. In another paper, Driffill and Sola (1998) applied the Markov switching model to justify whether there is an intrinsic bubble in stock prices so that stock prices deviate too far from the values predicted by the common predicted models: present value model or the fundamental asset evaluation using income and asset value. The paper concluded that a Markov switching model is a more appropriate representation of dividends because the dividends switch between regimes while stock prices can be better explained than through the bubble hypothesis.

In attempt to characterize the business environment and the switching between different regimes, Asea and Blomberg (1998) studied the lending behavior of banks over lending cycles, using the Markov switching model with a panel data set consisting of approximately two million commercial and industrial loans granted by 580 banks between 1977 and 1993. They demonstrated that banks change their lending standards from tightness to laxity systematically over the cycle.

The changing pattern of interest rates is indicative of business cycle conditions and could be subject to regime shifts itself. To investigate how real interest rates shift, Bekdache (1998) adopted a time varying parameter model with Markov switching conditional heteroscedasticity to capture two sources of shifts in real interest rates: shifts in coefficients and shifts in variance. The former relates the ex-ante real rate to the nominal rate, the inflation rate, and a supply shock variable, while the latter has unconditional shifts in the variance of the stochastic process. The results support a time varying parameter model over Markov switching with limited states. Dewachter (1996) studied interest rate volatility by examining both regime shifts in the variance and links between volatility and levels of the interest rate. While regime shifts were found in the variance, the contribution of volatility-level links cannot be ignored. The above findings suggest that univariate or single element regime shifts in interest rate modelling fail to fully characterize interest rate dynamics.

Research conducted by Kim and Nelson (1999) classifies the economy into two states of booms and recessions, then investigates whether there has been a structural break in post-war US real GDP growth towards stabilization. They used a Bayesian approach to identify a structural break at an unknown change-point in a Markov-switching model. Their empirical results suggested a break in GDP growth toward stabilization at the first quarter of 1984, and a narrowing gap between growth rates during recessions and booms. In 2000, Maheu and McCurdy used a Markov switching model to classify returns into a high-return stable state and a low-return volatile state. The two states bull and bear markets are respectively defined in the research question. The paper's finding is that although bull markets have a declining hazard function, the best market gains come at the start of a bull market. The paper also finds that volatility increases with duration in bear markets, which is intuitively foreseeable.

\subsubsection{Literature of the Field From 2000 to Present}

After the year 2000, the Hidden Markov models featured issues concerning mixture modeling. In 'A Markov switching model for annual hydrologic time series' by Akintug and Rasmussen (2005), the study assumes that the climate is switching between $\mathrm{M}$ states and that the state sequence can be described by a Markov chain. Observations are assumed to be drawn from a normal distribution whose parameters depend on the state variable. The paper presented stochastic properties of this class of models along with procedures for model identification and parameter estimation. There are strong similarities between MS and ARMA models, as those are applied to the time series of the mean annual discharge of the Niagara River. Although it is difficult to draw any general conclusion from a single case study, it appears that MS models (and ARMA models derived from MS models) generally have stronger auto-correlation at higher lags than ARMA models estimated by conventional maximum likelihood.

Marco Bazzi et al., (2014) proposed a new Markov switching model with time varying probabilities for the transitions. The novelty of the model is that the transition probabilities evolve over time by means of an observation driven model. The innovation of the time varying probability was generated by the score of the predictive likelihood function. This paper also studied the dynamic mean and variance behavior of U.S. Industrial Production growth. The findings include empirical evidence of changes in the regime switching probabilities, with more persistence for high volatility regimes in the earlier part of the sample. 
N. Sopipan et al (2014) proved that the MRS-GARCH model is the best performing model for gold price volatility according to some loss functions. The paper forecasts closing prices of gold price to trade future contracts using data from 2007 to 2011 in the Thai market.

Shih-Tang Hwuy et al. (2016) proposed a novel N state Markov-switching regression model in which the state indicator variable is correlated with the regression disturbance term. The model admits a wide variety of patterns for this correlation, while maintaining computational feasibility. The paper considered two applications of the $\mathrm{N}$ regime endogenous switching model, one to an empirical model of U.S. business cycles, and the other to a switching volatility model of U.S. equity returns.

Peter Nystrup et al. (2017) presented an adaptive estimation approach that allows for the parameters of the estimated models to be time varying. It was shown that a two state Gaussian hidden Markov model with time varying parameters can reproduce the long memory of squared daily returns that was previously believed to be the most difficult aspect to reproduce with a hidden Markov model. Capturing the time varying behavior of the parameters also leads to improved one step density forecasts.

Yoosoon Chang et al. (2017) introduced a new approach to model regime switching using an auto-regressive latent factor, which determines regimes depending upon whether it takes a value above or below some threshold level. The paper developed a modified Markov switching filter to estimate the mean and volatility models with Markov switching that are frequently analyzed and find that the presence of endogeneity in regime switching is indeed strong and ubiquitous.

Maciej Augustyniak, Luc Bauwens \& Arnaud Dufays (2019) proposed the factorial hidden Markov volatility (FHMV) process to model financial returns or realized variances. Its dynamics are driven by a latent volatility process specified as a product of three components: a Markov chain controlling volatility persistence, an independent discrete process capable of generating jumps in the volatility, and a predictable (data-driven) process capturing the leverage effect.

In summary, the Markov switching methodology was introduced by the seminal work of Hamilton (1989). Before the year 2000, Markov regime-switching was directly applicable to time series analysis in economics and finance for its dynamic nature. After the year 2000 there were significant studies to extend the scope of its applications, methodologies, and enhance its forecasting abilities. Even though Markov regime-switching has been well studied theoretically, the application and analysis in commodities prices likely need more development and combination with other disciplines: Financial Engineering, Trading in International Exchanges, and Behavioral Economics. There may be more fruitful outcomes in such collaboration works.

\section{Forecasting Models \& Results}

Extensive multi-disciplinary efforts have been spent on building a reliable model to forecast gold price. They include: The Economic Explanatory Model, Autoregressive Integrated Moving Average (ARIMA), Adaptive Neuro-Fuzzy Inference System (ANFIS), Multilayer Perceptron (MLP) Neural Network, Radial Basis Function (RBF) Neural Network, and Generalized Regression Neural Networks (GRNN). In the scope of our study, we develop the application of Markov regime switching in auto-regression models, and the performance of the model is evaluated. Our proposed MS auto-regression model will be based on components of the explanatory and auto-regression models; therefore, we apply these two models first to obtain the necessary foundation for comparison. 


\subsection{The Explanatory Model}

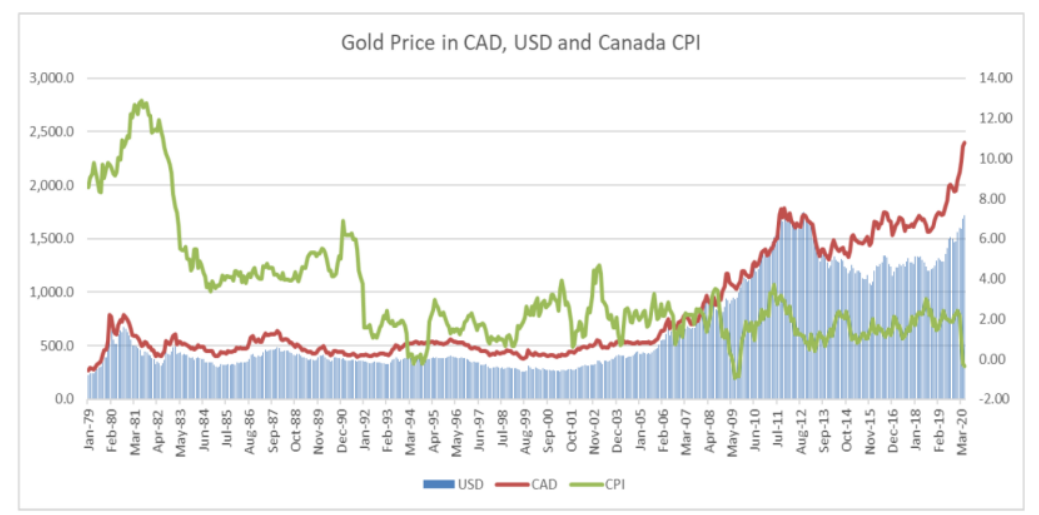

Figure 3. Gold price in CAD, USD, and the CPI change rate

We begin by looking at the time series of gold price in Canadian dollars. Details about the datasets are described in the appendix A. Figure 3 demonstrates the monthly average gold price from Jan. 1979 to May 2020. Intuitively it can be seen that an auto-regressive model would not yield a rational forecasting result because the impacts of economic factors are totally ignored. But the price in the previous month could provide a clue as to performance in the following month with a certain range of errors. Monthly average price is calculated by the total monthly revenue, divided by the quantity traded in the whole month. Using the monthly average price helps excluding the temporary shocks or short-term tendencies of the market.

The gold price in Canadian dollar can be explained by its price in USD, a major currency in the world economy, and the change in CPI. Therefore, it is modeled as a linear function of price in USD and the rate of change of CPI in the Canadian economy. We can always consider one or many other exogenous variables using a similar approach. Collinearity is an issue when using USD price as predictor, but that is a matter of fact; we could not reject its impacts on the gold price in Canada and must analyze the price in USD as it is the most powerful currency globally.

$$
\hat{y}_{t}=\alpha+\beta_{1} x_{1 t}+\beta_{2} x_{2 t}+\varepsilon_{t}
$$

where $\hat{y}_{t}$ is the forecasting spot price of gold at time $t$ in CAD, $x_{1 t}, x_{2 t}$ is the price in USD and CPI change rate at time $t$, respectively, $\varepsilon_{t}$ is a random term, or forecasting residual which is normally distributed. In this model, $x_{1 t}, x_{2 t}$ are exogenous variables; $\alpha, \beta_{1}, \beta_{2}$ are the coefficients that need to be estimated.

The regression of $\hat{y}_{t}$ with explanatory variables $x_{1 t}, x_{2 t}$ yields the outcome shown as in Table 1 . Note the significant codes are $0 * * * ; 0.001 * * ; 0.01 * ; 0.05$.; 0.11 .

Table 1. Fitted parameters of the Explanatory Regression Model

\begin{tabular}{lllll}
\hline Coefficients & Estimate & Std. Error & $\mathrm{t}$ value & $\operatorname{Pr}(>|\mathrm{t}|)$ \\
\hline Intercept & 112.56754 & 12.07728 & 9.321 & $<2 \mathrm{e}-16^{* * *}$ \\
USD & 1.07277 & 0.01167 & 91.911 & $<2 \mathrm{e}-16 * * *$ \\
CPI & -6.72388 & 1.80322 & -3.729 & $0.000215 * * *$
\end{tabular}

\begin{tabular}{|c|c|c|c|c|c|}
\hline Residuals & Min & 1Q & Median & $3 \mathrm{Q}$ & $\operatorname{Max}$ \\
\hline Value & -277.93 & -42.2 & 2.13 & 28.93 & 445.81 \\
\hline \multicolumn{3}{|c|}{ Residual standard error: } & \multicolumn{3}{|c|}{108.5 on 494 degrees of freedom } \\
\hline \multicolumn{3}{|c|}{ Multiple R-squared: } & \multicolumn{3}{|c|}{0.951} \\
\hline \multicolumn{3}{|c|}{ Adjusted R-squared: } & \multicolumn{3}{|c|}{0.9508} \\
\hline \multicolumn{3}{|c|}{$\begin{array}{l}\text { F-statistic: } \\
\text { p-value. }\end{array}$} & \multicolumn{3}{|c|}{$\begin{array}{l}4792 \text { on } 2 \text { and } 494 \mathrm{DF} \\
<2.2 \mathrm{e}-16\end{array}$} \\
\hline
\end{tabular}


This implies the explanatory model of gold price can be written as

$$
\hat{y}_{t}=112.56754+1.07277 x_{1 t}-6.72388 x_{2 t}+\varepsilon_{t}
$$

The negative coefficient of $x_{2 t}$ implies that CPI increases negatively impact on the gold price as we can roughly see in the Figure 3 above.

The R-squared value, which is a statistical measure, represents the proportion of the variance for a dependent variable that is explained by independent variables, takes a very high values, 0.951 . Therefore, we can say that the price can be well explained through the USD value and the CPI change. The two exogenous variables are good candidates for our MS auto-regression model, which will be presented later in subsection 3.3. Notably, we can see that the residuals may not be normally distributed (the quartile values are $-277.93 ;-42.23 ; 2.13 ; 28.93$; 445.81), this fact encourages us to explore a new forecasting model.

\subsection{The Auto-regression Model}

Gold price is an auto-regressive process. The price depends on 'itself' based on the previous time periods, with the residuals normally distributed.

$$
\hat{y}_{t}=\alpha+\sum_{i=1}^{p} \gamma_{i} y_{t-i}+\varepsilon_{t}
$$

where $\hat{y}_{t}$ is the forecasting spot price of gold at time $t, y_{t-i}$ is the gold price at time $t-i, \alpha, \gamma_{i}$ are parameters that need to be estimated, $p$ is the lag, $i \in[1, p]$, and $\varepsilon_{t}$ is a random term, or forecasting residual which is normally distributed. The outcome of the auto-regression in $\mathrm{R}$ gives

Table 2. Fitted Parameters of the Auto-regression Model, AR(1)

\begin{tabular}{llc}
\hline & AR1 & Intercept \\
\hline Coefficients: & 0.9994 & 826.7293 \\
S.E. & 0.0011 & 1223.7952 \\
\hline$\sigma^{2}$ estimated as 1381: & LL $=-2505.31$, & AIC $=5016.61$ \\
\hline
\end{tabular}

Using lags $p=2,3,4$ for the auto-regression model yields similar AIC values (4994.58, 4993.25, 4995 respectively). Therefore, we choose $\operatorname{AR}(1)$ with the lag $p=1$ for simplification, because the higher $p$ does not improve significantly forecast ability. This reflects our intuition and feature of the Markov chain, that the current state of the process only depends on the state of immediately preceding period. Table 3 below shows the outcome of AR(4) model with similar AIC.

Table 3. Fitted Parameters of the Auto-regression Model, AR(4)

\begin{tabular}{lllllc}
\hline & AR1 & AR2 & AR3 & AR4 & Intercept \\
\hline Coefficients: & 1.2355 & -0.3234 & 0.1030 & -0.0158 & 834.4876 \\
S.E. & 0.0449 & 0.0715 & 0.0716 & 0.0457 & 1118.6860 \\
\hline$\sigma^{2}$ estimated as 1306: & \multicolumn{3}{c}{$\mathrm{L}=-2491.57$} & AIC $=4995.13$ \\
\hline
\end{tabular}

The outcome of the model implies $y_{t}=(1-0.9994) * 826.7293+0.9994 y_{t-1}$. Hence, the auto-regression model is

$$
\hat{y}_{t}=0.4960+0.9994 y_{t-1}+\varepsilon_{t}
$$

\subsection{The MS Auto-regression Model}

Suppose that gold price is a random process following two regimes: in one regime, gold price depends only on an exogenous variables; in another regime, gold price is an auto-regressive process, where the price at time $t$ depends on the price at time $t-1$. This means that we impose both of the two models above and evaluate the switch between them described by a Markov process.

The model

$$
\hat{y}_{t}=\sum_{i=1}^{2} I_{i} \vec{\theta}_{i} x_{i}+\varepsilon_{t}
$$


where $\vec{\theta}_{i}$ is the parameters' vetor of the regime $i, i \in[1,2]$

$$
\vec{\theta}=\left[\theta_{1} \theta_{2} \theta_{3} \theta_{4}\right]
$$

and $\boldsymbol{x}_{\boldsymbol{i}}$ is the predictors' vector of the regime $i$

$$
\boldsymbol{x}=\left[\begin{array}{llll}
1 & x_{1} & x_{2} & y_{t-1}
\end{array}\right]^{T}
$$

where $I_{i}$ takes value 1 if the process is in regime $i, 0$ otherwise, and $\varepsilon_{t}$ is the residual term at time $t$ as usual. The Markov regime-switching model yields the following coefficients and statistics in Table 4.

\begin{tabular}{|c|c|c|c|c|c|c|}
\hline \multicolumn{7}{|l|}{ REGIME 1} \\
\hline & & Coefficients & Estimate & Std. Error & $\mathrm{t}$ value & $\operatorname{Pr}(>|t|)$ \\
\hline \multicolumn{2}{|c|}{ Residual standard error: 52.59953} & $($ Intercept $)(\mathrm{S})$ & 3.423 & 15.4871 & 0.221 & 0.825092 \\
\hline \multirow[t]{3}{*}{ Multiple R-squared: } & 0.9883 & $\mathrm{USD}(\mathrm{S})$ & 0.0918 & 0.0282 & 3.2553 & $0.001133 * *$ \\
\hline & & $\mathrm{CPI}(\mathrm{S})$ & 1.5468 & 1.7671 & 0.8753 & 0.381411 \\
\hline & & CAD1(S) & 0.9265 & 0.0239 & 38.7657 & $<2.2 \mathrm{e}-16 * * *$ \\
\hline \multicolumn{2}{|c|}{ Standardized Residuals } & Min & $1 \mathrm{Q}$ & Median & $3 Q$ & Max \\
\hline \multicolumn{2}{|l|}{ Value } & -139.4999 & -5.4652 & -0.3805 & 0.6528 & 203.4511 \\
\hline \multicolumn{7}{|c|}{ REGIME 2} \\
\hline \multirow{2}{*}{\multicolumn{2}{|c|}{ Residual standard error: 12.88244}} & Coefficients & Estimate & Std. Error & $\mathrm{t}$ value & $\operatorname{Pr}(>|t|)$ \\
\hline & & $($ Intercept $)(\mathrm{S})$ & 44.4512 & 4.2992 & 10.3394 & $<2.2 \mathrm{e}-16 * * *$ \\
\hline \multirow[t]{3}{*}{ Multiple R-squared: } & 0.9962 & $\mathrm{USD}(\mathrm{S})$ & 0.1998 & 0.0235 & 8.5021 & $<2.2 \mathrm{e}-16 * * *$ \\
\hline & & $\mathrm{CPI}(\mathrm{S})$ & -2.1165 & 0.3151 & -6.7169 & $1.856 \mathrm{e}-11 * * *$ \\
\hline & & CAD1(S) & 0.7676 & 0.0249 & 30.8273 & $<2.2 \mathrm{e}-16 * * *$ \\
\hline \multicolumn{2}{|c|}{ Standardized Residuals } & Min & 1Q & Median & $3 \mathrm{Q}$ & $\operatorname{Max}$ \\
\hline \multicolumn{2}{|l|}{ Value } & -32.0437 & -3.87 & 0.0138 & 5.2002 & 35.7343 \\
\hline \multicolumn{7}{|c|}{ INFORMATION CRITERIA FOR } \\
\hline \multicolumn{7}{|l|}{ BOTH REGIMES } \\
\hline & & \multicolumn{2}{|l|}{ AIC } & \multicolumn{2}{|l|}{$\mathrm{BIC}$} & LL \\
\hline & & \multicolumn{2}{|l|}{4611.161} & \multicolumn{2}{|l|}{4694.466} & -2297.58 \\
\hline
\end{tabular}

Table 4. Fitted parameters of the MS Auto-regression Model, MS AR(1)

The equations for the two regimes, equation 20 represents Regime 1 and equation 21 represents Regime 2 :

$$
\begin{aligned}
& \hat{y}_{t 1}=3.4230+0.0918 x_{1}+1.5468 x_{2}+0.9265 y_{t-1} \\
& \hat{y}_{t 2}=44.4512+0.1998 x_{1}-2.1165 x_{2}+0.7676 y_{t-1}
\end{aligned}
$$

The Markov-switching model has both regimes significant, where the R-squared values are 0.9883 and 0.9962.

Equation 16 describes the negative impact of CPI change on gold price in the explanatory model. But in 20 and 21 we observe the more sophisticated effects of the hidden mechanism that controls this impact. In some periods of time the CPI change has positive marginal effects on gold price. 
Regime 1

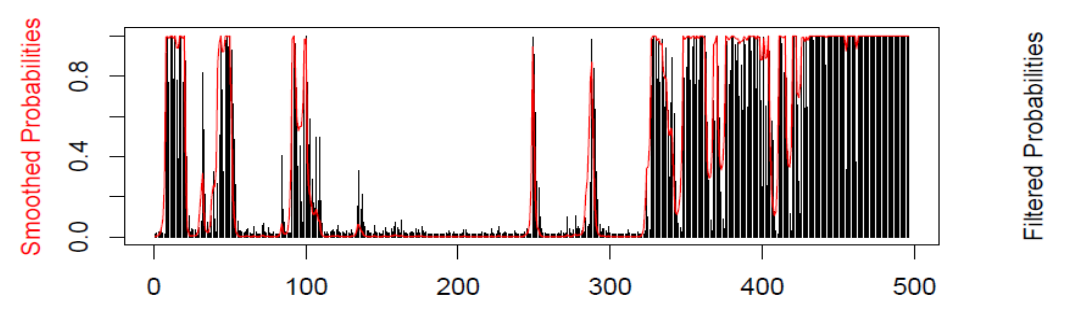

Regime 2

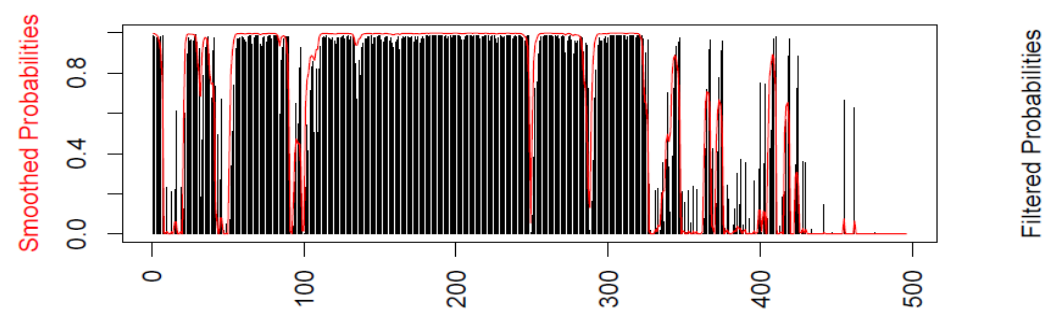

Figure 4. Graph of filtered and smoothed probabilities

Table 5 at the end of this section shows the transition probability matrix: the transition probability matrix has high determinant values, which indicates that it is difficult to change from one regime to the other. The model perfectly detects the periods of each state. The residuals are white noise and show a better fit to the normal distribution in each regime than in the explanatory model (see Table 1).

Figure 4 above describes filtered and smoothed probabilities. Since the smooth probabilities are evaluated using the whole dataset while the filtered probability at time $t$ are evaluated using data up to and including time $t$ (but excluding time $t+1, \ldots, T$ ), we can realize a slight difference between them.

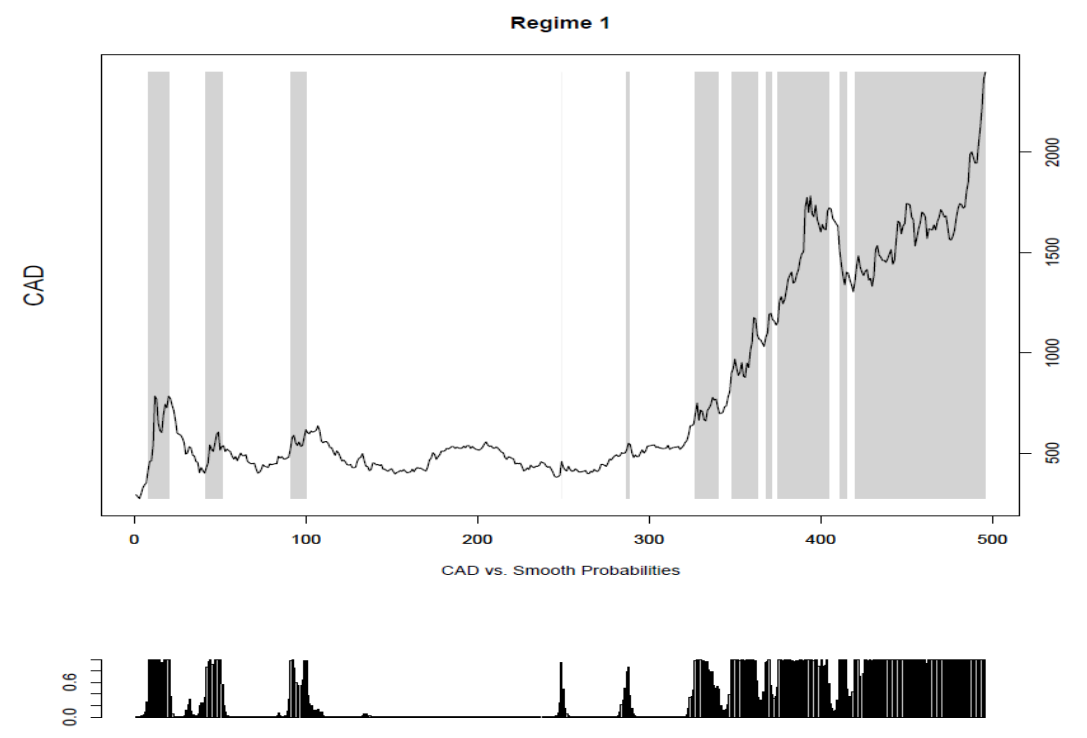

Figure 5. Graph of price vs. smoothed probabilities

In Figure 5, the regimes and price are represented in the same graph. It shows that almost all the substantial increases of gold price are associated with regime 1. This implies that the abnormal increases of, or the shocks in gold price, resulted from special economic conditions. 


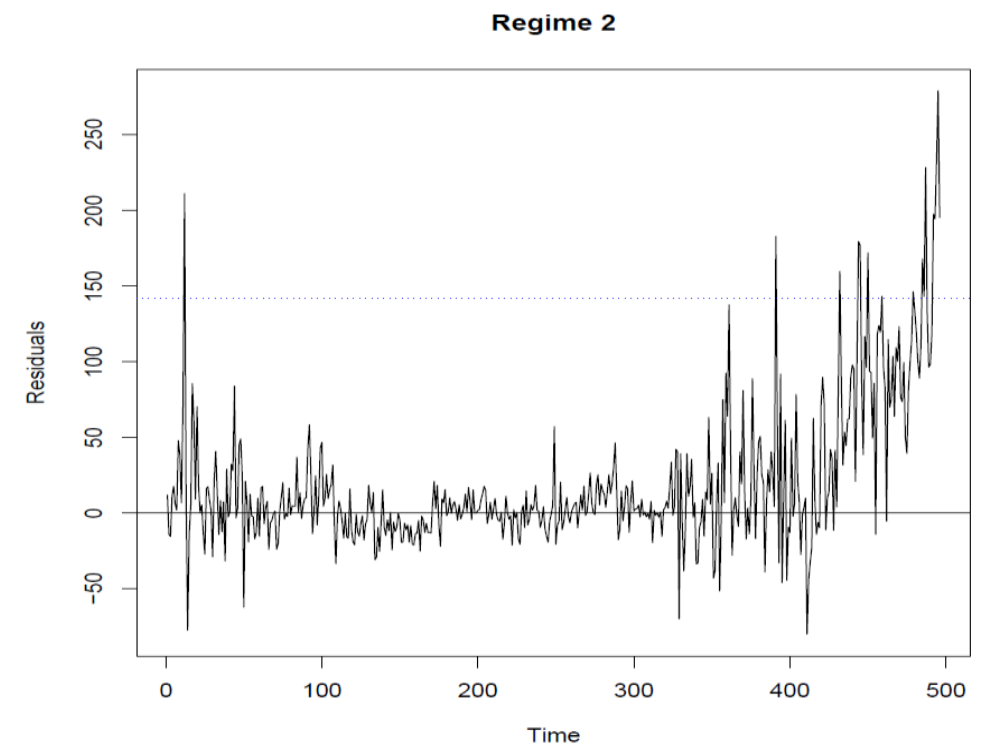

Figure 6. Graph of Residuals, upper panel: regime 1, lower panel: regime 2

Figure 6, the upper panel introduces the residual terms of the model with regime 1. It reveals, for example, in the periods from Jul. 2005 to May 2020 (observations from 330 to 497), gold price follows regime 1, and the residual is a white noise. The lower panel describes the residual terms of the model with regime 2 . It can be clearly seen that, in the period from Jul. 2005 to May 2020 (observations from 330 to 497), the residual has a tendency with a mean greater than 0 ; it is a non-stationary process. This confirms that the price follows regime 1. In Figure 7 below we combine both two regimes in the model, the graph of residual terms fluctuates around zero. Visibly it looks like white noise. The calculation confirms that the mean value of the residuals is $1.9744 \times 10-8 \approx 0$.

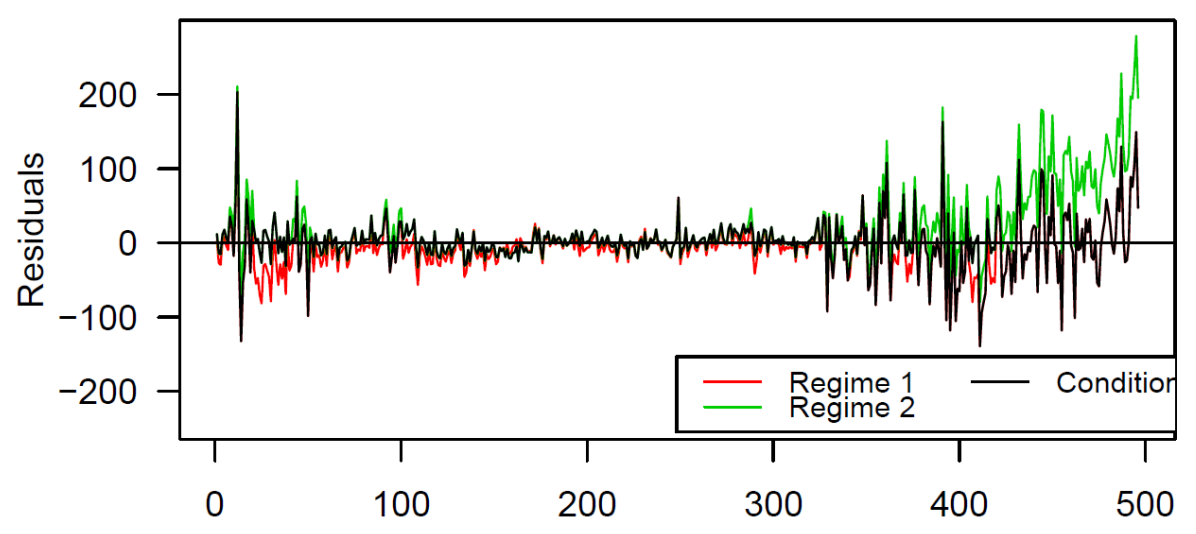

Figure 7. Graph of residuals, the two regimes 
Table 5. Transition Probability Matrix P(2,2)

\begin{tabular}{ccl}
\hline Regime 1 & Regime 2 \\
Regime 1 0.93419448 & 0.06580552 \\
Regime 2 0.03776094 & 0.96223906 \\
\hline
\end{tabular}

\subsection{Forecasting Work}

Suppose that this month, gold price is in regime 1, probability that next month it will stay in regime 1 is $93.42 \%$ and visit regime 2 is $6.58 \%$. Probability that gold price process stays in regime 2 for the two consecutive months is $3.78 \%$ while probability that it moves from regime 2 to regime 1 is $96.22 \%$.

The dataset used as sample is from Jan. 1979 to May 2020. Now given that the spot price in USD for Jun. 2020 is 1732.2, the CPI change rate is 0.5472, the price in CAD in May 2020 is 2398.9. Using equations 20 and 21 obtains

$$
\begin{gathered}
\hat{y}_{t 1}=3.4230+0.09181732 .2+1.54680 .5472+0.92652398 .9=2385.887 \\
\hat{y}_{t 2}=44.4512+0.19981732 .2-2.11650 .5472+0.76762398 .9=2230.794
\end{gathered}
$$

The graph in Figure 5 shows that, May 2020 the gold price is in regime 1. Using the transition probability matrix $\mathrm{P}(2,2)$ above yields the expected price in June $\hat{y}_{t}=0.93422385 .887+0.06582230 .794=2375.672$

The actual price of gold in June, according to World Gold Council (Note 5) is CAD 2347.5 per ounce. The forecasting error is $\left|1-\frac{2375.672}{2347.5}\right| \approx 1.198 \%$. Similarly, we use the formula 1 to obtain the $k$-step transition probability, then forecasting for $k$ months ahead $\hat{y}_{t_{0}+k}$.

\subsection{Evaluation of Models' Performance}

The most popular performance measure for forecasting models is the mean squared-error. There are two variants of this measure: Mean Absolute Percentage Error (MAPE) and Root Mean Square Error (RMSE).

The Mean Absolute Percentage Error defined as

$$
\text { MAPE }=\frac{\sum N \frac{\left|\hat{y}_{t}-y_{t}\right|}{y_{t}}}{N}
$$

where $\hat{y}_{t}, y_{t}$ are the forecasted price and actual price at time $t$, respectively. By our calculation, the auto-regression model AR(1) has an MAPE of $2.9862 \%$. The explanatory model yields an MAPE of $7.3063 \%$. Using the MS auto-regression model we obtain a forecasting error average of $2.6085 \%$.

Using the Root Mean Square Error (RMSE) measure

$$
\text { RMSE }=\sqrt{\frac{\sum N\left(\hat{y}_{t}-y_{t}\right)^{2}}{N}}
$$

our proposed MS auto-regression model has RMSE of 33.6233, one of the best performance among the models evaluated in literature (R. Hafezi et al. 2018).

\section{Conclusion and Potential Research}

In this research, we studied the theoretical background of the Markov chain Regime-switching model, and its application in forecasting data series. The paper proposed a Markov Regime-switching auto-regression model for forecasting gold price. The results showed that the MS auto-regression model is very efficient in the analysis of historical data series, specifically, gold price in the Canadian market. The specific application of Markov regime-switching in forecasting gold price with two regimes was examined and compared with other popular analyzing methods. We showed that the MS auto-regression performs dominantly. The forecasting model for gold price in two regimes was proposed.

The R-squared values in the two regimes of the model are 0.9883 and 0.9962. Using Mean Absolute Percentage Error to evaluate the forecasting error, using the MS auto-regression model we can obtain the forecasting error average of $2.6085 \%$. This is a significant improvement in comparison with other popular models in the literature. The model with 
exogenous variables gives MAPE $=7.3063 \%$ and the model with auto-regression yields MAPE $=2.9862 \%$. The Transition Probability Matrix, which is considered as an important property of the specific Markov chain, was evaluated. Applying the forecating model for a specific moment of time, we obtained a forecating price in June 2020 with error less than $1.2 \%$.

We argued and presented statistical evidence that the dramatic changes in gold price likely happen in regime 1 which is explained by macroeconomic factors. Socio-economic conditions substantially impact gold price. The other commodity price series can be analyzed using the same method. We can also partition a long period of time into many regimes by giving $N \geq 2$ or applying $N=2$ multiple times.

The forecasted price series fits very well with the actual gold price series as per the Figure 8 .

\subsection{Potential Research}

We know that, when the transition matrix $\mathbf{P}$ for a Markov chain is regular, then the Markov chain has a unique limit vector (known as a steady-state vector), regardless of the values of the initial probability vector by the property of the Markov chain. If the transition matrix $\mathbf{P}$ for a Markov chain is regular, the positive powers of $\mathbf{P}$ approach a limit (which is a matrix) all of whose columns equal the chain's steady-state vector. This might be particularly beneficial to some type of commodity prices or asset returns (including stock prices) because then we will have a steady state, meaning stable prices over time.

Suppose a transition probability matrix $\mathbf{P}$, initial state vector $\vec{v}_{0}$, steady state vector $\vec{v}_{\infty}$; I denotes the identity matrix. After $k$ periods, the state vector is:

$$
\vec{v}_{0} P^{k}=\vec{v}_{\infty}
$$

Solving the following equation yields the steady state vector $\vec{v}_{\infty}$.

$$
\vec{v}_{\infty}(P-I)=0
$$

The steady state is quite possible in reality when the demand and supply of a commodity are saturated in the market. In a business and legal environment, a study in historical data series can address the time and the conditions where entries to an industry would be stable.

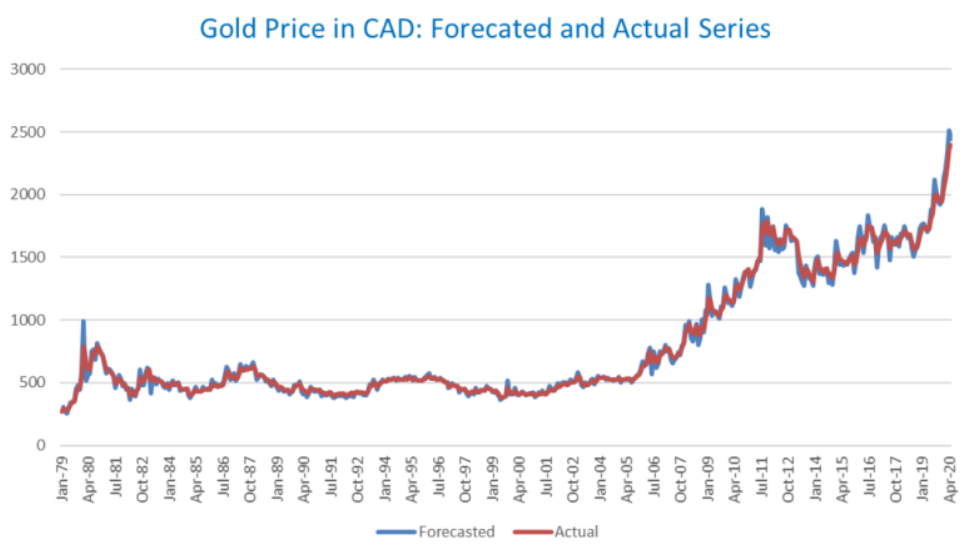

Figure 8. Gold price in CAD - Forecasted and actual series

In federal or provincial administration, the tax debts have significant impacts on government budgeting and public policies. Studies in steady-states of these processes would benefit the economy and social welfare by figuring out the circumstances that there would be no large deviation in tax collection and hence, there would be no more struggling with budget deficit.

\section{Acknowledgments}

This research was supported, in part, by an NSERC Discovery Research Grant and by Carleton University. We would like to express our sincere thanks to Snezana Vucic for inspiring and help in implementation of this research, Patrick Boiley and Song Cai for valuable comments, and Peter Wesolowski for proofreading the manuscript. 


\section{References}

Akintug, B., \& Rasmussen, P. F. (2005). A Markov switching model for annual hydrologic time series. Water Resources, 41, W09424. https://doi.org/10.1029/2004WR003605

Asea, P. K., \& Blomberg, S. (1998). Lending cycles. Journal of Econometrics, 83(1-2), 89-128. https://doi.org/10.1016/S0304-4076(97)00066-3

Bekdache, B. (1998). Alternative Approaches to Modeling Time Variation in the Case of the U.S. Real Interest Rate. Computational Economics, 11(1-2), 41-51.

Chang, Y., et al.. (2017). A new approach to model regime switching. Journal of Econometrics, 196.

David, A., et al.. (2017, October). Markov-Switching GARCH Models in R: The MSGARCH Package. Journal of Statistical Software, 91(4). https://doi.org/10.18637/jss.v091.i04

Dewachter, H. (1996). Modelling interest rate volatility: Regime switches and level links. Weltwirtschaftliches Archiv, 132, 236258. https://doi.org/10.1007/BF02707806

Hafezi, R., \& Akhavan, A. N. (2018). Forecasting Gold Price Changes: Application of an Equipped Artificial.

Hamilton, J. D. (1989). A New Approach to the Economic Analysis of Nonstationary Time Series and the Business Cycle. Econometrica, 57(2), 357-384. https://doi.org/10.2307/1912559

Hwuy, S-T., et al.. (2016). N-State Endogenous Markov-Switching Models. Working paper, Department of Economics, University of Washington.

John, M., Maheu, T., \& McCurdy, H. (2000). Identifying Bull and Bear Markets in Stock Returns. Journal of Business Economic Statistics, 18(1), 100-112. https://doi.org/10.1080/07350015.2000.10524851

Kim, C.-J., \& Nelson, C. (1999). Has the U.S. Economy Become More Stable? A Bayesian Approach Based on A Markov-switching Model of The Business Cycle. The Review of Economics and Statistics, 81(4), 608-616.

Maciej, A., \& Luc, B. A. D. (2019). A New Approach to Volatility Modeling: The Factorial Hidden Markov Volatility Model. Journal of Business Economic Statistics, 37(4). https://doi.org/10.1080/07350015.2017.1415910

Marco, B. et al.. (2017). Time Varying Transition Probabilities for Markov Regime Switching Models. Journal of Time Series Analysis.

Neural Network. AUT J. Model. Simul. Eng., 50(1), 71-82. https://doi.org/10.22060/miscj.2018.13508.5074

Nguyen, B. A., \& Aggey, S. (2015). Trends of Base Metals Prices. Theoretical Economics Letters, 5, 531-540. https://doi.org/10.4236/tel.2015.54062

Organization for Economic Co-operation and Development. Consumer Price Index: Total All Items for Canada [CPALTT01CAM659N]. FRED, Federal Reserve Bank of St. Louis. Retrieved July 21, 2020, from https://fred.stlouisfed.org/series/CPALTT01CAM659N

Peter, N., Henrik, M., \& Erik, L. (2017, December). Long Memory of Financial Time Series and Hidden Markov Models with Time Varying Parameters. Journal of Forecasting, 36(8), 989-1002.

Robert, H. S., \& David, S. S. (2017). Time Series Analysis and Its Applications With R Examples. Springer International Publishing.

Sola, M., \& Driffill, E. (1994). Testing the term structure of interest rates using a stationary vector autoregression with regime switching. Journal of Economic Dynamics and Control, 18(3-4), 601-628.

Sopipan, N., Sattayatham, P., \& Premanode, B. (2014). Forecasting Volatility of Gold Price Using Markov Regime Switching and Trading Strategy. Journal of Mathematical Finance, 2(1).

Wang, P. (2002). Financial Econometrics. London: Routledge. https://doi.org/10.4324/9780203892879

\section{Notes}

Note 1. Nguyen Bao Anh \& Aggey Semenov, 2015

Note 2. https://www.gold-eagle.com/forecasts-predictions

Note 3. Data: World Gold Council. https//www.gold.org/goldhub

Note 4. Data: Yahoo Finance. https://ca.finance.yahoo.com

Note 5. https://www.gold.org/goldhub/data/gold-prices 


\section{Appendix A}

\section{Abbreviation}

AIC

AR

$\mathrm{ARCH}$

ARIMAX

BIC

CPI

GARCH Generalized Autoregressive Conditional Heteroskedasticity

HMM

LBMA

LL

MAPE

MLE

MS

MSM

RMSE
Akaike Information Criterion

Autoregression (Model)

Autoregressive Conditional Heteroskedasticity

Auto Regressive Integrated Moving Average with eXogeneous Input

Bayesian Information Criterion

Consumer Price Index

Hidden Markov Model

London Bullion Market Association

Log Likelihood function

Mean Absolute Percentage Error

Maximum Likelihood Estimate

Markov-Switching

Markov-Switching Model

Root Mean Square Error (RMSE)

\section{Appendix B}

SYMBOLS

$\begin{array}{ll}\alpha, \beta_{1}, \beta_{2}, \gamma_{i} & \text { Coefficients of the models } \\ \varepsilon_{t} & \text { Residuals, random, or irregular terms } \\ f\left(y_{t} \mid y_{t-1}\right) & \text { Probability density function of } y_{t}, \text { given } y_{t-1}, 2 \text { states } \\ g\left(y_{t} \mid \Omega_{t-1}\right) & \text { Probability density function of } y_{t} \text {, given information at } t \text { - } 1, \text { N-states } \\ L(\vec{\theta}) & \text { Likelihood function of parameters } \\ \Omega_{t} & \text { Information available at time } t \text {, gold price model } \\ p_{i j} & \text { The transition probability from state } i \text { to } j \\ \mathbf{P} & \text { Transition Probability Matrix } \\ s_{t} & \text { State at time } t \text { (value) } \\ S_{t} & \text { State at time } t \text { (variable) } \\ \sigma^{2} & \text { Variance of the data at time } t \\ \vec{\theta}_{i} & \text { The parameters' vector of the model } \\ \boldsymbol{x} & \text { The predictors' vector of the model, including the lags if any } \\ y_{t} & \text { Gold price at time } t \text {, also known as information available at time } t \\ \hat{y}_{t} & \text { Forecasting price at time } t\end{array}$

\section{Copyrights}

Copyright for this article is retained by the author(s), with first publication rights granted to the journal.

This is an open-access article distributed under the terms and conditions of the Creative Commons Attribution license (http://creativecommons.org/licenses/by/4.0/). 